\title{
Implosion-Induced Collapse Effect of Initial Penetration Damage on Concrete Structures with Finite Thickness
}

\author{
Hao Geng $\mathbb{D},{ }^{1,2}$ Hao Lu $\mathbb{D},{ }^{1}$ Songlin Yue, ${ }^{1}$ Ziming Xiong $\mathbb{D},{ }^{1}$ Shanzheng Sun, ${ }^{1}$ Mu Huang, \\ and Zhongzheng Jiang ${ }^{1}$ \\ ${ }^{1}$ State Key Laboratory of Disaster Prevention and Mitigation of Explosion and Impact, Army Engineering University of PLA, \\ Nanjing 210007, China \\ ${ }^{2}$ The No. $95979^{\text {th }}$ Troop of PLA, Taian 271000, China
}

Correspondence should be addressed to Hao Lu; lh829829@163.com

Received 26 March 2020; Revised 4 June 2020; Accepted 6 July 2020; Published 29 July 2020

Academic Editor: Michele Brun

Copyright (c) 2020 Hao Geng et al. This is an open access article distributed under the Creative Commons Attribution License, which permits unrestricted use, distribution, and reproduction in any medium, provided the original work is properly cited.

Attacks using penetration-explosion warheads can cause the backside of concrete targets to collapse. Current research on collapse damage mainly focuses on the damage caused by external explosions and the damage of targets with prefabricated holes under internal blasts. However, the penetration effect also affects the destructive effect of the explosion-induced collapse, so experiments on prefabricated borehole charges cannot fully reflect real implosions. To study the effect of the initial penetration damage of imploding concrete structures with finite thickness on their collapse, in this study, field experiments are carried out on concrete targets with different initial penetration damage. The collapse damage of the targets is analyzed, and the dimensional analysis method is used to fit the experimental data and obtain the rules and prediction methods of the effect of the initial penetration damage on the relative collapse thickness. The collapse depth of the concrete targets is found to decrease with the increase in the impact energy factor; this increase is found to reduce gradually until stabilization. The conclusion has been verified and analyzed in depth through numerical simulations. The results of this study can provide a basis for subsequent simulations of the actual penetration and explosion effects and a reference for the optimal protection design of concrete structures and the optimal damage design of penetration-explosion warheads.

\section{Introduction}

Penetration-explosion warheads are currently the first choice of weapon against concrete targets. They rely on the kinetic energy or jets to penetrate a certain depth inside the target and detonate high-energy charges to damage the target. Warheads damage concrete structures of finite thickness in the area near the impact point. They may produce an impact or blast funnel pit on the front side and a collapse funnel pit on the back side [1]. In severe cases, penetrating damage may occur. Depending on the destructive effect, the collapse caused by the warhead to the structure can be categorized into impact collapse and explosion collapse [2].

Collapse is an important form of damage to concrete structures caused by earth penetrator weapons. After the warhead hits the target, under the effect of explosion and (or) impact, the concrete structure with finite thickness will experience collapse damage on its back side. When the compressive stress wave generated by the explosion load (or impact load) propagates to the back of the structure, a strong tensile wave is generated, which causes the concrete on the back of the structure to collapse and spallate, forming concrete fragments of different sizes. These concrete fragments have a high speed and can hurt personnel and damage equipment [3].

The first discovery of collapse was observed by Hopkinson during a scientific study of the explosion effect in 1914, but real attention toward this issue was given only after the 1950s. Some scientific research institutions, military departments, companies, and universities have obtained a series of empirical formulas for the thickness of collapses by 
summarizing a large number of test data during World War II and later. These formulas can be divided into three categories: the first category involves penetration depth of the projectile, the second category involves the penetration thickness, and the third category includes the empirical formulas built directly from collapse experimental data. Specifically, the first category includes Petry-I formula, Petry-II formula, ACE formula, National Defence Research Committee (NDRC) formula, modified NDRC formula, and Kar formula. The second category includes BRL formula, modified BRL formula, Ammann-Whitney formula, and PCDM and its modified formulas. The third category of empirical formulas includes Haldar formula, Hughes formula, Adeli-Amin formula, Bechtel formula, Stone-Webster formula, and Chang formula [2].

The purpose of this study is to discuss the collapse damage caused by an explosion, so we need to focus on the third category of empirical formulas. In 1981, Chang proceeded from the perspective of kinetic energy and energy and used Bayesian statistical analysis to obtain the following semiempirical formula for calculating the thickness of collapse:

$$
h=0.118 \frac{m^{0.4} v^{0.67}}{D^{1.2} \sigma_{c}^{0.4}}
$$

where $m$ is the projectile mass, $v$ is the initial penetration velocity, $D$ is the projectile diameter, and $\sigma_{c}$ is the compressive strength of the target material.

There is very little public information available about experimental research on explosion-induced collapse. The empirical formula for the thickness of projectile explosioninduced collapse is only mentioned in the Protective Construction Design Manual (PCDM) published by the US Air Force in 1989 [4]. The manual uses the following empirical formula:

$$
h=0.048\left(\frac{R}{W^{1 / 3}}\right)^{-0.3}\left(\frac{W}{W+C}\right)^{-0.3} W^{1 / 3},
$$

where $W$ is the weight of the projectile charge, $C$ is the weight of the projectile shell, and $R$ is the distance from the projectile to the structure during the explosion. Zheng et al. pointed out that equation (2) is conservative and the calculation of the collapse failure curve calculated by it contains a lot of experimental data without collapse [2]. Therefore, based on the experiment data, they used the reliability design method to modify equation (2) and proposed a new formula for calculating the collapse thickness:

$$
h=0.0115\left(\frac{R}{W^{1 / 3}}\right)^{-0.7}\left(\frac{W}{W+C}\right)^{-1.31} W^{1 / 3} .
$$

Recent research on the collapse damage of concrete structures under explosion mainly focused on two aspects: the collapse damage of the backside of concrete targets under external explosion and the collapse damage of the backside of concrete targets with precast holes under internal explosion. Fu and Zhang [5] studied the damage to the free surface of a reinforced concrete target subjected to an internal explosion load; $\mathrm{Li}$ and Hao [6] studied the damage mode for reinforced concrete slabs under aerial initiation conditions; and Zhang and Wang [7] studied the antiexplosion performance of concrete gravity dams under the impact of underwater explosion shocks. Rabczuk et al. [8] used field tests and numerical simulations on concrete slabs of different thicknesses and initiated explosive contact to compare the diameters and depths of craters on the front and back of concrete targets. Leng et al. [9] studied the dynamic response of cavity formation and developed predictive rules for concrete structure under the effect of internal explosion load, and Lai et al. [10] studied the failure characteristics of super-strength concrete under different burial depths and different loading conditions. Zhang et al. [11] studied the critical collapse thickness and the corresponding critical charge for a concrete target. Rabczuk et al. $[8,12]$ conducted field tests and numerical simulations on concrete slabs of different thicknesses and initiated explosive contact to compare the diameters and depths of the craters on the front and back of the concrete targets.

In fact, most of the explosive damage is investigated under the premise that the structure experiences initial penetration damage; i.e., the warhead produces penetration damage to the front of the concrete structure first. The explosion venting effect produced by the front penetration pit reduces the explosion energy on the back surface, thereby reducing the collapse damage of the back side. At present, research on the impact of the initial penetration damage on the collapse of imploding concrete targets with finite thickness is scarce. In this study, we experimentally investigate the damage of the back of a finite-thickness concrete structure under different initial damage conditions and column-type charges. By fitting the experimental data, we obtain an empirical formula that describes the implosioninduced collapse considering the initial penetration damage is obtained.

\section{Theoretical Analysis}

Collapse damage involves a series of complex mechanisms, such as wave propagation effects in the thickness direction of the concrete structures and transient dynamic effects of materials, which are theoretical problems that need to be solved urgently in the field of protection engineering. Since the middle of the last century, scientists from various countries have made great efforts to understand the collapse effect theoretically. For example, in a series of experiments of the detonation of contacting explosives and metal plates, multiple spalling phenomena were observed. Based on this experiment, in this study, the detonation wave is reduced to a one-dimensional saw-shaped wave (no step-up triangular load) and the waveform change and attenuation during the wave propagation process is not considered. Principal stress theory was used to determine the conditions for spalling caused by the wave reflection on the back surface, the number of spalling fragments, and the thickness and velocity of each fragment. Principal stress theory can also be used to determine the thickness of the one-dimensional spallation and the velocity of the fragments under the action of a stepped triangular load. Based on preliminary experiments, 
it can be concluded that the thickness of the collapse and the velocity of the diaphragm are related to the shape of the carrier, time of action, peak pressure, tensile strength and density of the material, and speed of wave propagation. These theoretical observations significantly simplify the materials into homogeneous brittle materials (one-dimensional) and homogeneous elastic materials but do not reflect the complex spatial, time, and cumulative effects of the collapse damage $[4,13]$.

To solve this issue, an empirical formula considering the effects of implosion-induced collapse was established, and the dimensional analysis method was used to simplify it and find the relationship between multiple physical quantities $[4,14,15]$. This is an effective and practical method to study the influencing factors and laws of the collapse damage of structures. In this study, the length-force-time (L-F-T) mechanical system is used to conduct a dimensional analysis of the collapse effect of concrete structures with finite thickness.

The destructive effect of implosion-reduced collapse is determined in terms of factors such as the amount, depth, diameter, and length of the charge and the parameters of the concrete material. Because the initial penetration damage is random and, thus, difficult to measure, the impact energy factor (dimensionless quantity) is used to represent the energy of the projectile on the target during penetration. In 1982, Haldar et al. analyzed from a dimensional perspective and pointed out that one end of the National Defence Research Committee (NDRC) formula proposed by the National Defence Research Committee in 1946 is dimensionless while the other end is dimensional $[2,16]$. To correct this, they introduced a dimensionless parameter called the impact energy factor:

$$
I=\frac{N m v^{2}}{D^{3} \sigma_{c}}
$$

where $N$ represents the shape factor of the warhead. This parameter can better indicate the damage status of the concrete target from the penetration effect and ignores the influence of the randomness.

The penetration and explosion effects acting on the concrete structure are essentially the process of the concrete material undergoing shear, compression, and stretching. The governing parameters involved in the implosion of concrete under initial penetration damage are shown in Table 1 [17-24].

From a physical point of view, the collapse depth depends on the material and geometric parameters of the explosive and the target and the degree of initial penetration damage [24-26], which can be summarized as

$$
h=f\left(\rho_{e}, l, d, s, I, E, \sigma_{c}, \sigma_{t}, \mu, \rho_{T}, H, v_{d}\right) .
$$

In accordance with the theory of material mechanics and explosion dynamics, Table 1 and equation (5) list the basic parameters involved in concrete materials under the implosion effect. When the structure is subjected to shear, tension, and compression, these parameters are variables that need to be investigated for different damage effects of
TABLE 1: Governing parameters of the implosion of concrete under initial penetration damage.

\begin{tabular}{lcc}
\hline Parameter & Symbol & Units \\
\hline Explosive density & $\rho_{e}$ & $\mathrm{FT}^{2} \mathrm{~L}^{-4}$ \\
Explosive length & $l$ & $\mathrm{~L}$ \\
Explosive diameter & $d$ & $\mathrm{~L}$ \\
Charge depth & $s$ & $\mathrm{~L}$ \\
Impact energy factor & $I$ & - \\
Concrete elastic modulus & $E$ & $\mathrm{FL}^{-2}$ \\
Concrete uniaxial compressive strength & $\sigma_{c}$ & $\mathrm{FL}^{-2}$ \\
Concrete uniaxial tensile strength & $\sigma_{t}$ & $\mathrm{FL}^{-2}$ \\
Concrete Poisson's ratio & $\mu$ & - \\
Concrete density & $\rho_{T}$ & $\mathrm{FT}^{2} \mathrm{~L}^{-4}$ \\
Concrete thickness & $H$ & $\mathrm{~L}$ \\
Explosive detonation velocity & $v_{d}$ & $\mathrm{FT}^{-1}$ \\
Collapse depth & $h$ & $\mathrm{~L}$ \\
\hline
\end{tabular}

the material (except the impact energy factor). Other parameters that are not listed herein can be converted from these parameters. Using the Buckingham theorem $[4,14,15]$, equation (5) can be expressed in the following dimensionless form:

$$
\frac{h}{d}=f\left(\frac{\rho_{e} v_{d}^{2}}{d^{2} E^{3}}, \frac{l}{d}, \frac{s}{d}, I, \frac{\sigma_{c}}{E}, \frac{\sigma_{t}}{E}, \mu_{T}, \frac{\rho_{T} v_{d}^{2}}{d^{2} E^{3}}, \frac{H}{d}\right) .
$$

\section{Experimental Setup}

In the experiment, a total of 20 concrete targets with a strength of C35 and dimensions of $1000 \mathrm{~mm} \times 500 \mathrm{~mm}$ were used. The concrete was fixed in the circumferential direction with an $8 \mathrm{~mm}$ steel hoop to eliminate the radial boundary effect, as shown in Figure 1. First, a penetration experiment was performed on ten targets at the field site to induce initial penetration damage. A $30 \mathrm{~mm}$ orbital smoothbore gun was used in the penetration experiment. Figure 2 shows a schematic of the experimental setup, and Figure 3 shows a photograph of the experiment site.

A $30 \mathrm{CrMnSiNi2A}$ solid projectile with a mass of $168 \mathrm{~g}$ and a material yield strength of $1413 \mathrm{MPa}$ was used. The projectile is difficult to remove after penetration into the target and affects the charge position of the explosion experiment. Therefore, the projectile was designed with a gradually decreasing diameter, as shown in Figure 4 . The length of the projectile was $100 \mathrm{~mm}$, and the diameters of the projectile neck and tail were 20 and $14 \mathrm{~mm}$, respectively. A groove was designed at the tail of the projectile to facilitate the use of a specially made flat head screwdriver to rotate the projectile after penetration. The warhead was oval-shaped and its caliber radius head was 2.5 . The initial penetration speed of the projectile was controlled at $460-670 \mathrm{~m} / \mathrm{s}$. After the penetration experiment and once the projectile was removed, the implosion experiment was performed.

The explosion experiment was performed in a static explosion field, and the Hexogen grain was pressed in advance (Figure 5) to a size of $20 \mathrm{~mm} \times 20 \mathrm{~mm}$. After penetration, we measured the ten targets and recorded their penetration depth. We drilled holes in the center of the other 


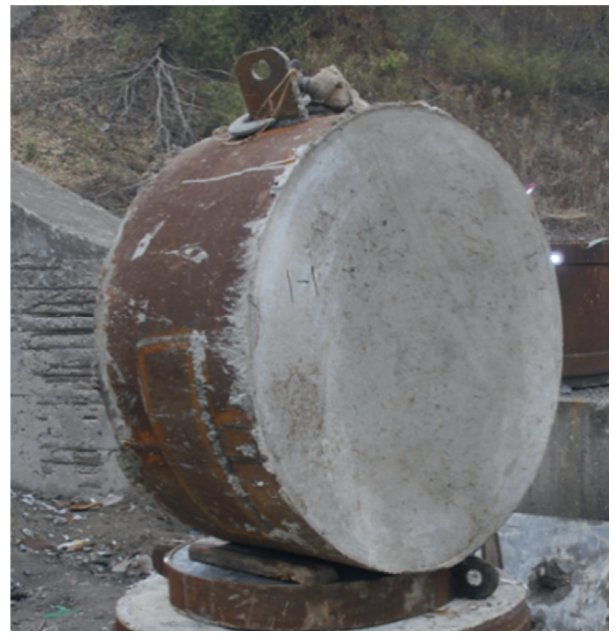

FIgURE 1: Experimental concrete target.

High-speed photography background board

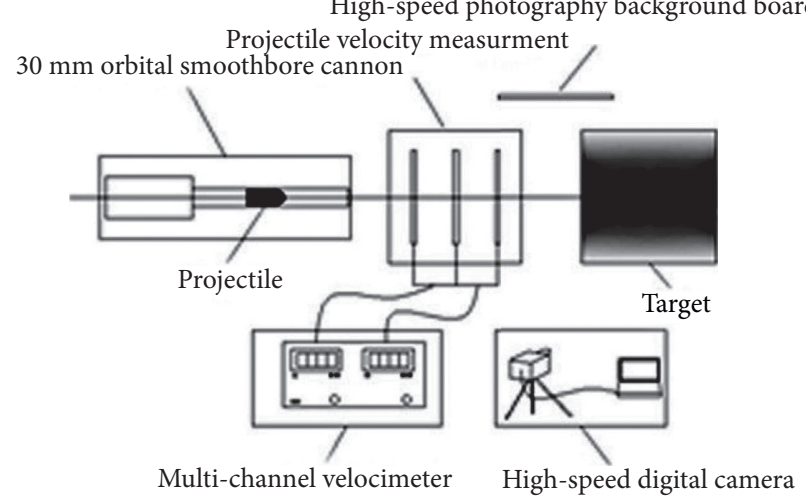

Figure 2: Penetration experiment schematic diagram.

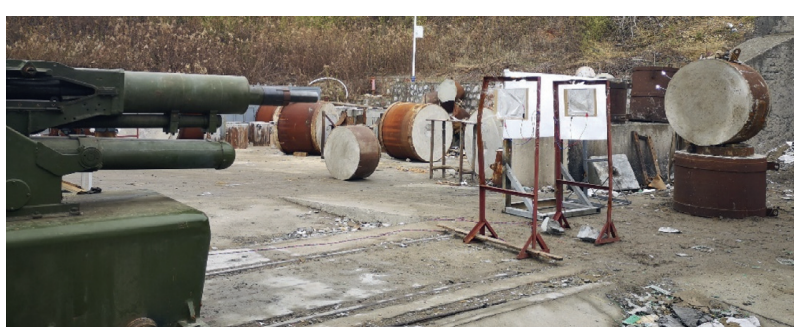

FIgure 3: Penetration experiment site.

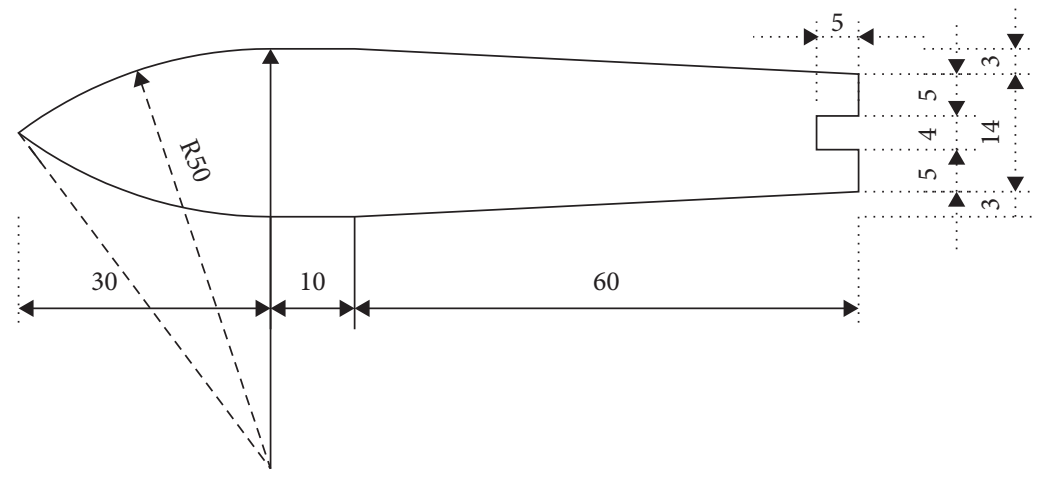

(a)

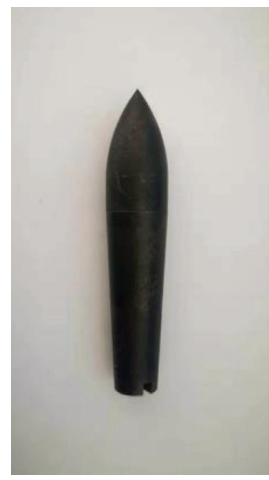

(b)

FIGURE 4: Schematic (a) and photograph (b) of the penetration projectile. 


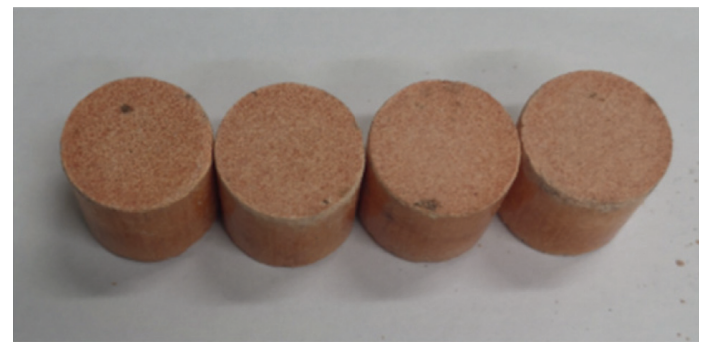

Figure 5: Pressed grain.

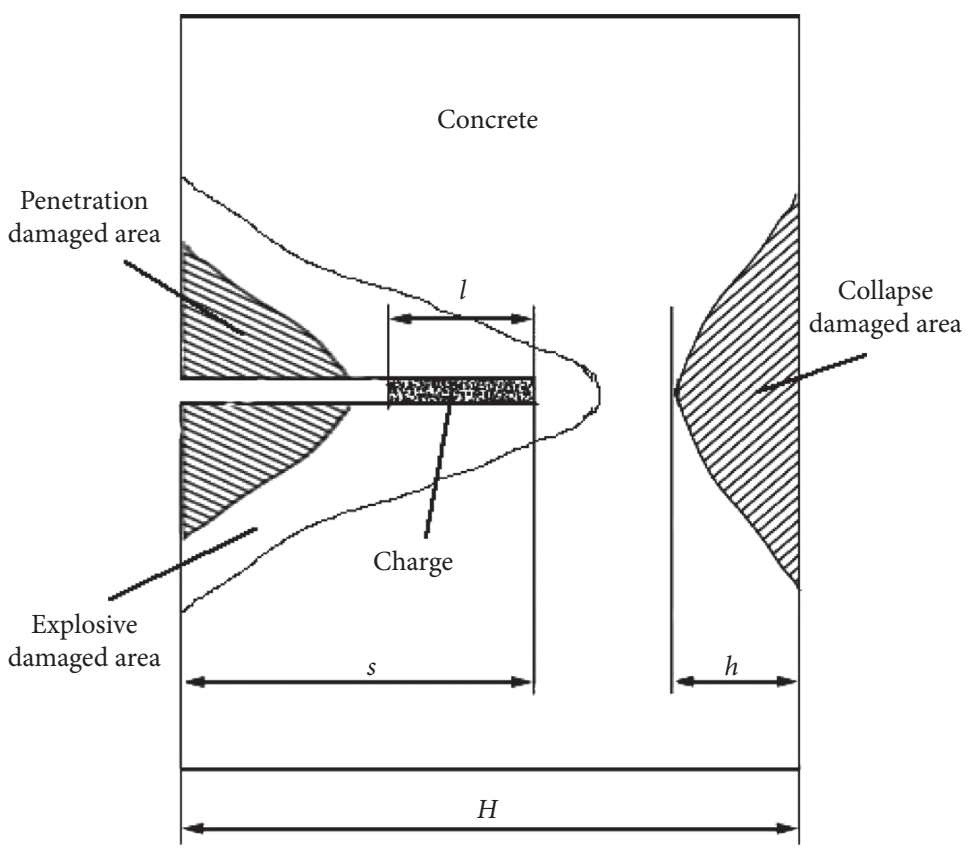

FIGURE 6: Cylindrical charge layout.

ten targets to a depth equal to the ten depths obtained from the penetration experiment. The explosive was arranged in the penetration tunnel, and the column charging method was adopted. The arrangement of the components of the experiments is shown in Figure 6. The explosive was passivated Hexogen with a density of $1.5 \mathrm{~g} / \mathrm{cm}^{3}$, explosion heat of $5215 \mathrm{~J} / \mathrm{g}$, detonation velocity of $8150 \mathrm{~m} / \mathrm{s}$, and charge diameter of $20 \mathrm{~mm}$.

It is worth noting that the setting of parameters in the experiment takes the actual engineering scenario into consideration. The size of the projectile/charge matches the target size, and the shrink ratios are the same. If the target is too large or the projectile/charge is too small, it is not in line with engineering reality, and its damage status is easier to judge. The destruction law of critical state is of additional research significance as it can also act as a reference for the optimal design of the layer resisting projectile or penetration-explosion warheads that exhibit the desired price-performance ratio.

\section{Results and Discussion}

To study the impact of the initial penetration damage on the implosion-induced collapse of concrete structures, a controlled variable method was adopted to divide 20 targets into ten groups for explosion experiments, as shown in Table 2. Then, the collapse thickness of the targets was compared. After the explosion, funnel-shaped collapse occurred on most surfaces, and a few cracks appeared outside the damage area. Partial cracks communicated with each other causing fragmentation in some areas, and part of the concrete was peeled off from the back of the targets. The collapse was obvious and the integrity of the target was poor after the explosion. Photography with a speed of 3000 frames/s was used to record the concrete throwing process during the explosion. Figure 7 shows the images of the concrete thrown at target 7-2 when subjected to an explosive load. After the experiment, the collapse damage of the targets was measured and recorded. Figure 8 shows the damage of the backside of some targets. A 3D scanner is used to record the damage on the target's back. The scan results are shown in Figure 9. Table 2 shows the relevant data of the target after the explosion.

We verified that the experimental results conform to the aforementioned authoritative empirical formulas. The shape, diameter, and mass of the projectile that caused the initial penetration damage, as well as the mass, density, and 
TABLE 2: Experimental results of collapse damage of concrete targets.

\begin{tabular}{|c|c|c|c|c|c|c|c|}
\hline Group & No. & Penetration speed $(\mathrm{m} / \mathrm{s})$ & Charge aspect ratio & Charge quality (g) & Impact factor & Buried depth $(\mathrm{m})$ & Collapse depth $(\mathrm{m})$ \\
\hline \multirow{2}{*}{1} & $1-1$ & 465.12 & $5: 1$ & 39.25 & 172.68 & 0.197 & 0.097 \\
\hline & $1-2$ & - & $5: 1$ & 39.25 & 0 & 0.197 & 0.152 \\
\hline \multirow{2}{*}{2} & $2-1$ & 489.39 & $5: 1$ & 39.25 & 193.38 & 0.214 & 0.091 \\
\hline & $2-2$ & - & $5: 1$ & 39.25 & 0 & 0.214 & 0.148 \\
\hline \multirow{2}{*}{3} & $3-1$ & 512.75 & $5: 1$ & 39.25 & 209.62 & 0.232 & 0 \\
\hline & $3-2$ & - & $5: 1$ & 39.25 & 0 & 0.232 & 0.142 \\
\hline \multirow{2}{*}{4} & $4-1$ & 544.35 & $5: 1$ & 39.25 & 235.93 & 0.251 & 0.098 \\
\hline & $4-2$ & - & $5: 1$ & 39.25 & 0 & 0.251 & 0.149 \\
\hline \multirow{2}{*}{5} & $5-1$ & 558.49 & $5: 1$ & 39.25 & 248.28 & 0.259 & 0.105 \\
\hline & $5-2$ & - & $5: 1$ & 39.25 & 0 & 0.259 & 0.154 \\
\hline \multirow{2}{*}{6} & $6-1$ & 589.43 & $5: 1$ & 39.25 & 277.31 & 0.277 & 0.105 \\
\hline & 6-2 & - & $5: 1$ & 39.25 & 0 & 0.277 & 0.149 \\
\hline \multirow{2}{*}{7} & $7-1$ & 612.92 & $5: 1$ & 39.25 & 300.29 & 0.287 & 0.125 \\
\hline & $7-2$ & - & $5: 1$ & 39.25 & 0 & 0.287 & 0.163 \\
\hline \multirow{2}{*}{8} & $8-1$ & 625.21 & $5: 1$ & 39.25 & 315.60 & 0.293 & 0.122 \\
\hline & $8-2$ & - & $5: 1$ & 39.25 & 0 & 0.293 & 0.158 \\
\hline \multirow{2}{*}{9} & $9-1$ & 643.47 & $5: 1$ & 39.25 & 330.49 & 0.310 & 0.114 \\
\hline & $9-2$ & - & $5: 1$ & 39.25 & 0 & 0.310 & 0.151 \\
\hline \multirow{2}{*}{10} & $10-1$ & 671.46 & $5: 1$ & 39.25 & 359.87 & 0.325 & 0.119 \\
\hline & $10-2$ & - & $5: 1$ & 39.25 & 0 & 0.325 & 0.154 \\
\hline
\end{tabular}

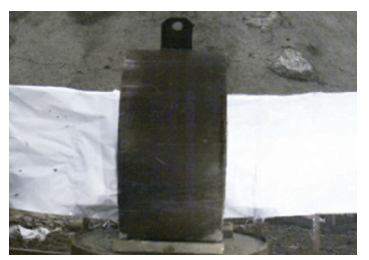

(a)

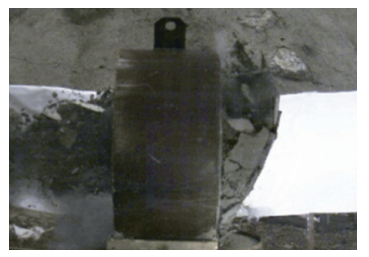

(e)

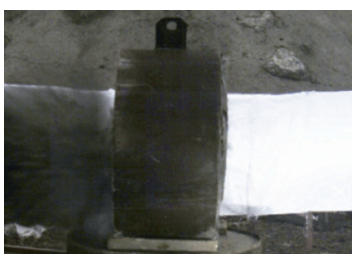

(b)

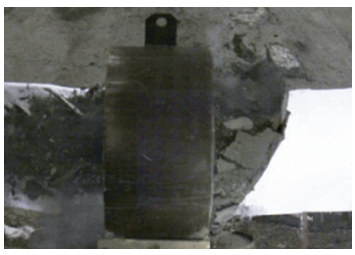

(f)

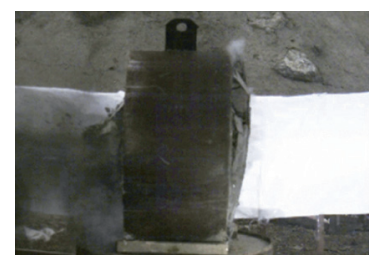

(c)

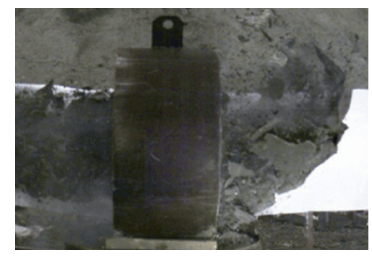

(g)

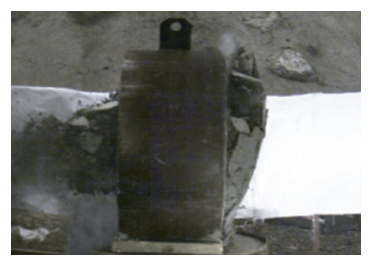

(d)

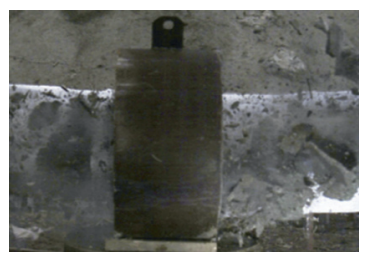

(h)

FiguRE 7: High-speed photography of the concrete throwing experiment with target 7-2 at time. (a) $t=0 \mathrm{~s}$, (b) $t=0.001 \mathrm{~s}$, (c) $t=0.0025 \mathrm{~s}$, (d) $t=0.01 \mathrm{~s}$, (e) $t=0.03 \mathrm{~s}$, (f) $t=0.075 \mathrm{~s}$, (g) $t=0.09 \mathrm{~s}$, and (h) $t=0.18 \mathrm{~s}$.

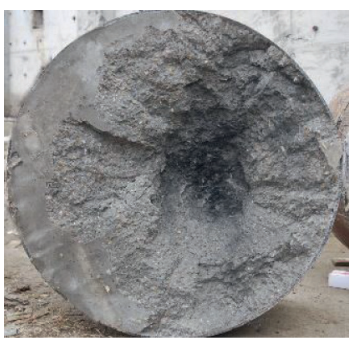

(a)

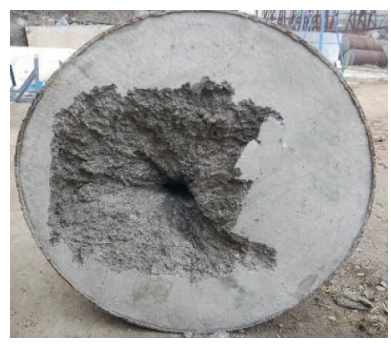

(b)

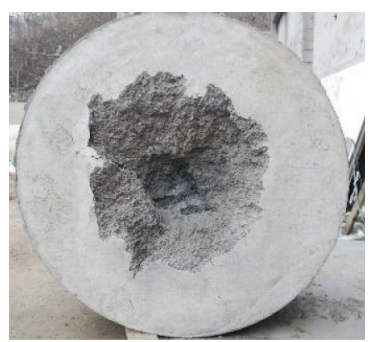

(c)

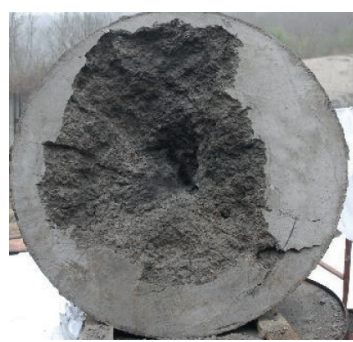

(d)

FIGURE 8: Collapse damage effect of concrete targets: (a) target 2-2; (b) target 5-1; (c) target 7-1; (d) target 8-2.

detonation speed of the cylindrical charge, were fixed. Therefore, the penetration parameters only retain the initial penetration speed, and the explosion parameters only retain the charge depth. For concrete materials, the elastic modulus, Poisson's ratio, and density are fixed values, and the thickness of the targets is also fixed. Therefore, the 


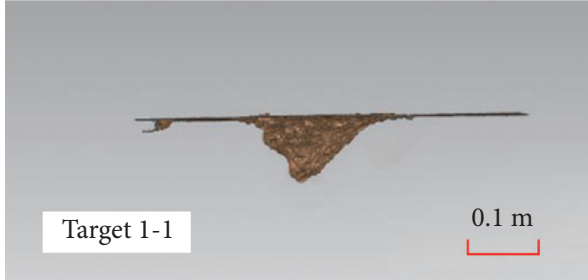

(a)

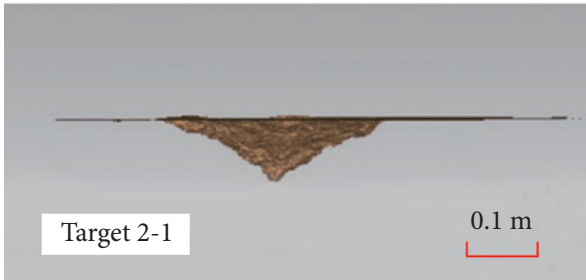

(c)

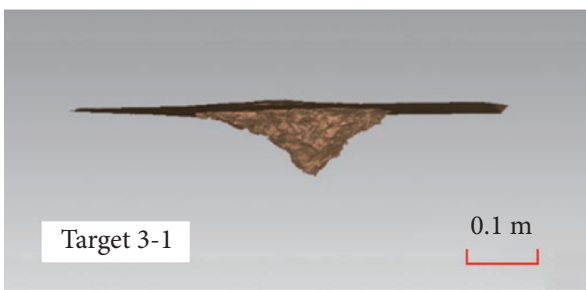

(e)

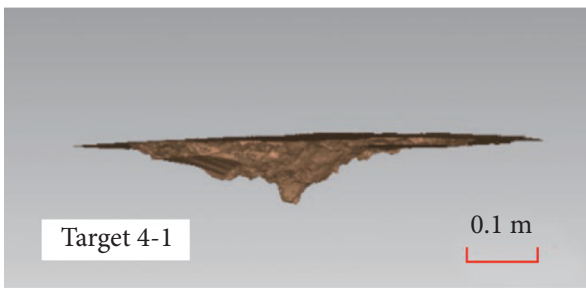

(g)

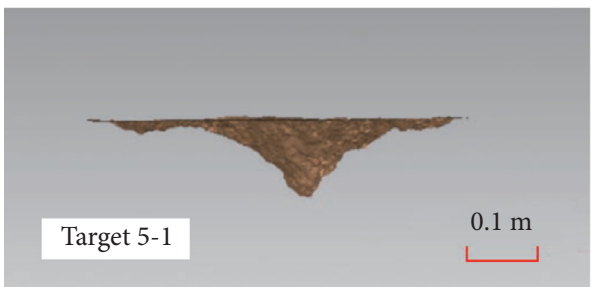

(i)

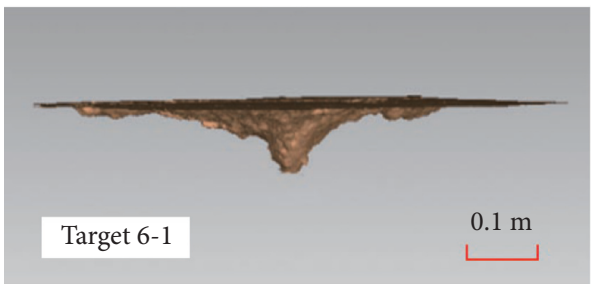

(k)

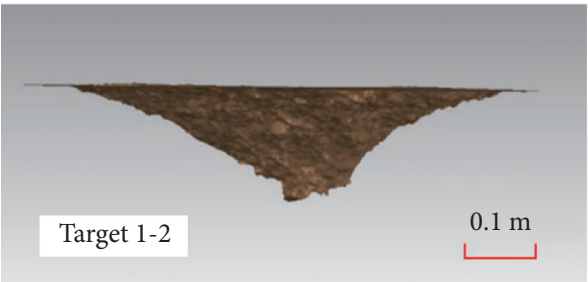

(b)

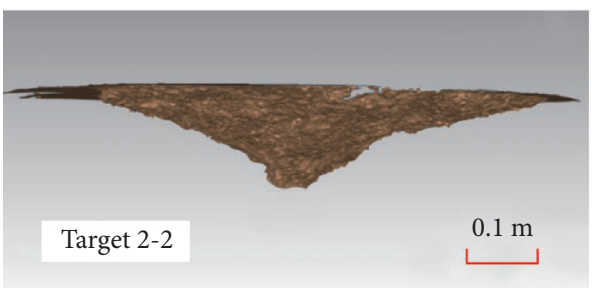

(d)

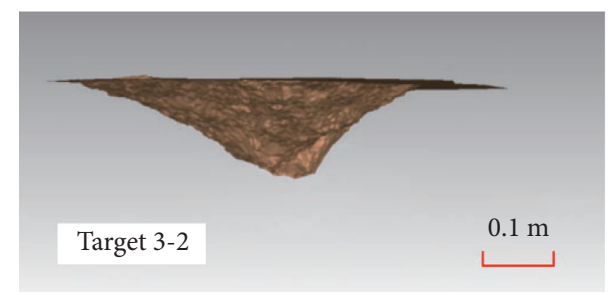

(f)

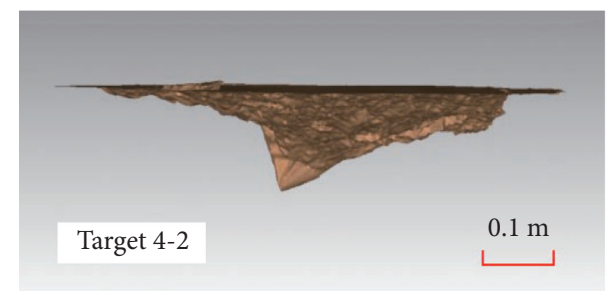

(h)

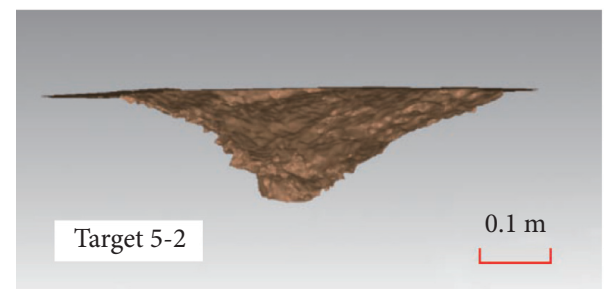

(j)

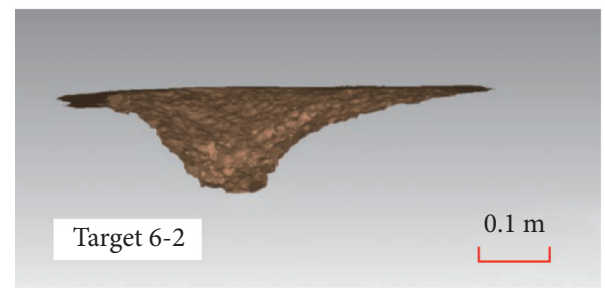

(l)

Figure 9: Continued. 


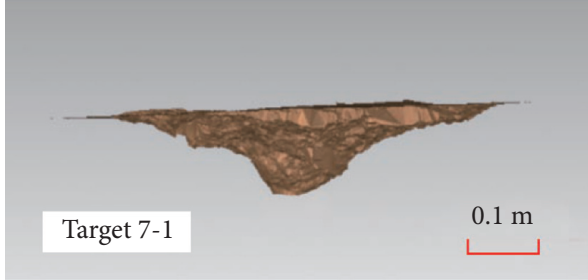

(m)

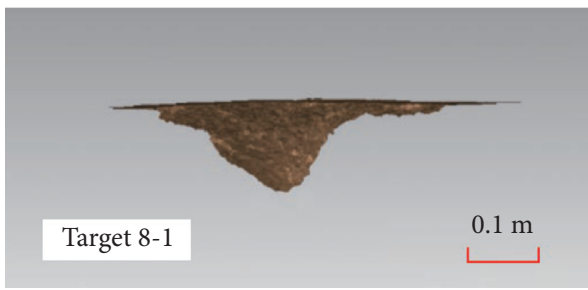

(o)

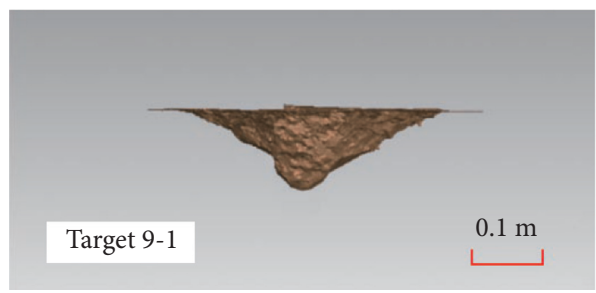

(q)

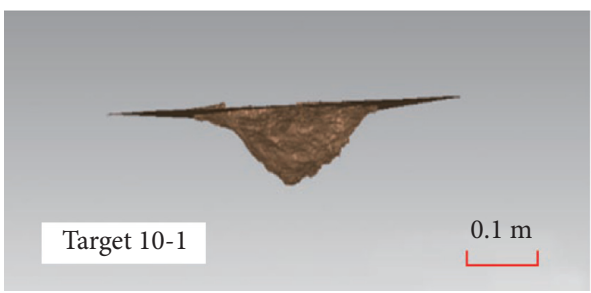

(s)

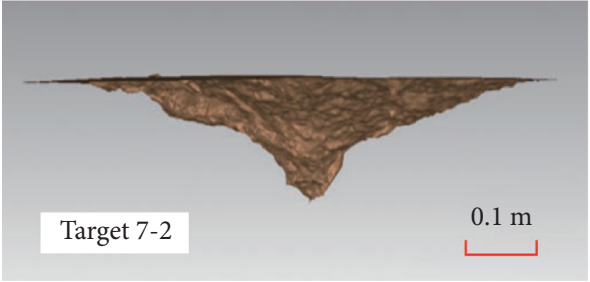

(n)

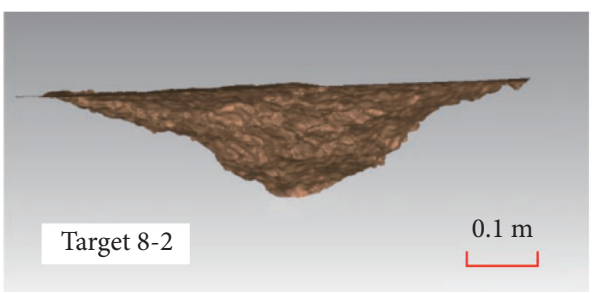

(p)

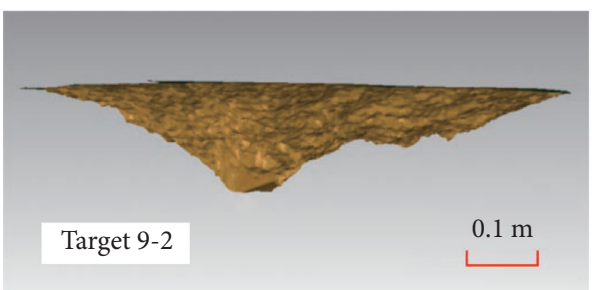

$(\mathrm{r})$

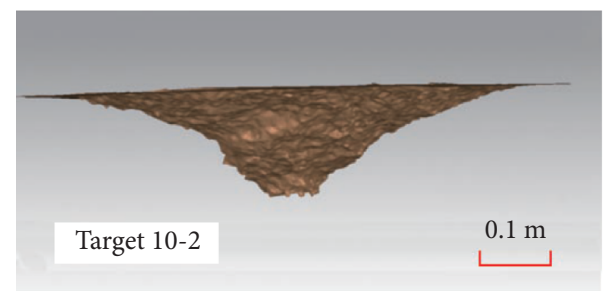

$(\mathrm{t})$

Figure 9: Scan images of the collapse damage.

dimensionless expression of the explosion-induced collapse depth of a finite-thickness concrete structure in the state of initial penetration damage can be simplified to

$$
\frac{h}{d}=f\left(\frac{s}{d}, I\right) .
$$

According to the existing empirical formula and massive test data [26], when the proportion of charge burial depth $(s-l) / m^{1 / 3}$ is greater than 0.2 ( $m$ is the charging mass), the completely packing coefficient is

$$
\overline{r_{p}}=1.65 K_{p} \sqrt[3]{m}
$$

where $K_{p}$ represents the medium failure yield coefficient. This formula shows that, for a certain medium material and charge, the completely packing coefficient is constant. In other words, the coefficient indicating the charging depth and packing condition is constant under this condition. In this experiment, the proportions of charge burial depth are all greater than 0.33 , so the influence of the charge depth on the explosion-induced collapse can be ignored. Equation (7) can be further simplified as

$$
\frac{h}{d}=f(I) \text {. }
$$

The dimensionless collapse depth obtained by substituting the experiment data is shown in Figure 10.

As can be seen from Figure 10, in the second group, the target with penetration damage did not form the collapse damage area after the explosion, and only cracks formed on the backside. To evaluate the effect of the penetration damage on the collapse damage area, the second set of data is removed during analysis, and only the remaining nine sets are analyzed. To study the impact of the penetration damage on the implosion-induced collapse effect, the difference between each set of experiment data is used to represent the magnitude of the impact, i.e., the dimensionless amount of the difference in collapse depth: 


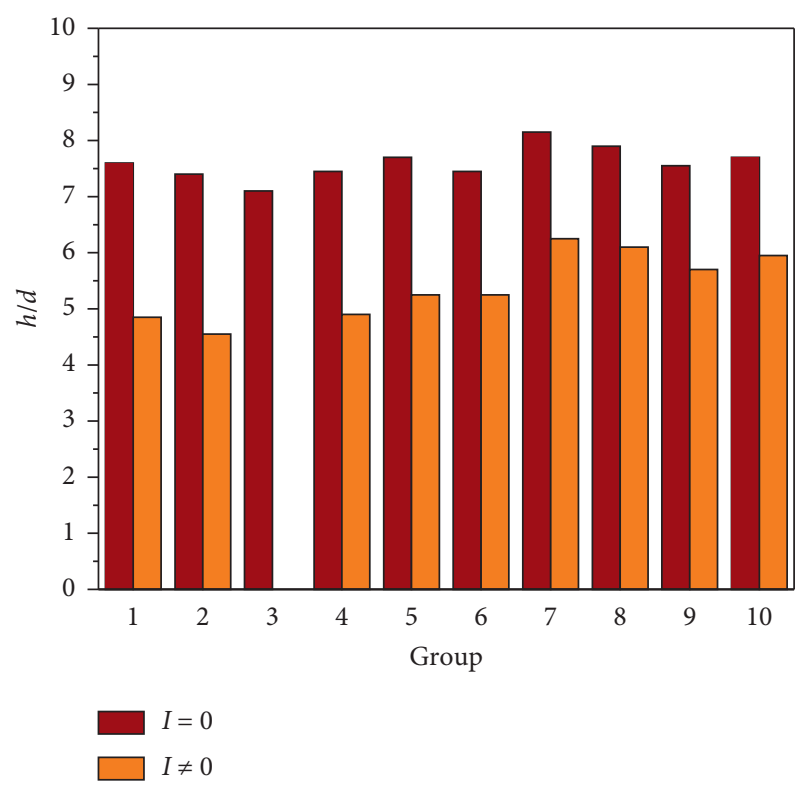

FIgURE 10: Experimental data of the dimensional collapse depth.

$$
\frac{h^{\prime}}{d}=\frac{h_{n 2}}{d}-\frac{h_{n 1}}{d}, \quad n=1,3,4, \ldots, 7,
$$

where $h_{n 2}$ and $h_{n 1}$ are the collapse thicknesses without and with penetration damage in the $n$ group experiment, respectively. The relationship between the dimensionless collapse depth and the impact energy factor is shown in Figure 11.

When the impact energy factor is small, the penetration of the damage area has a greater impact on the escape of the explosion energy due to the shallow penetration depth. Therefore, the difference in the collapse depth is large, which may lead to the failure of collapse due to excessive energy dissipation. As the impact energy factor gradually increases, the proportion of the penetration tunnel area in the entire damage area gradually increases, and the escape energy gradually decreases. The difference in collapse depth in the same group of experiments gradually decreases. When the impact energy factor increases to a certain value, the impact of the penetration damage area on the explosion energy gradually stabilizes, and the difference between the collapse depths decreases. Therefore, equation (9) can be expressed as a logistic function:

$$
\frac{h^{\prime}}{d}=f(I)=\frac{1}{\alpha+\beta \times a^{I-b}}+k .
$$

In equation (11), $\alpha>0$ and $\beta>0$, with $a \neq 1, b, k$, are constants, which are fitted through experimental data by using least squares approach in MATLAB to obtain

$$
\frac{h^{\prime}}{d}=\frac{1}{0.9187+0.6129 \times 1.043^{I-255.8}}+1.734,
$$

where $I$ is between 170 and 360. Figure 11 shows the results of the fitting of equation (12) to the experimental results. As shown in Figure 12, equation (12) fits the experimental results well, and the uncertainty does not exceed $5 \%$.

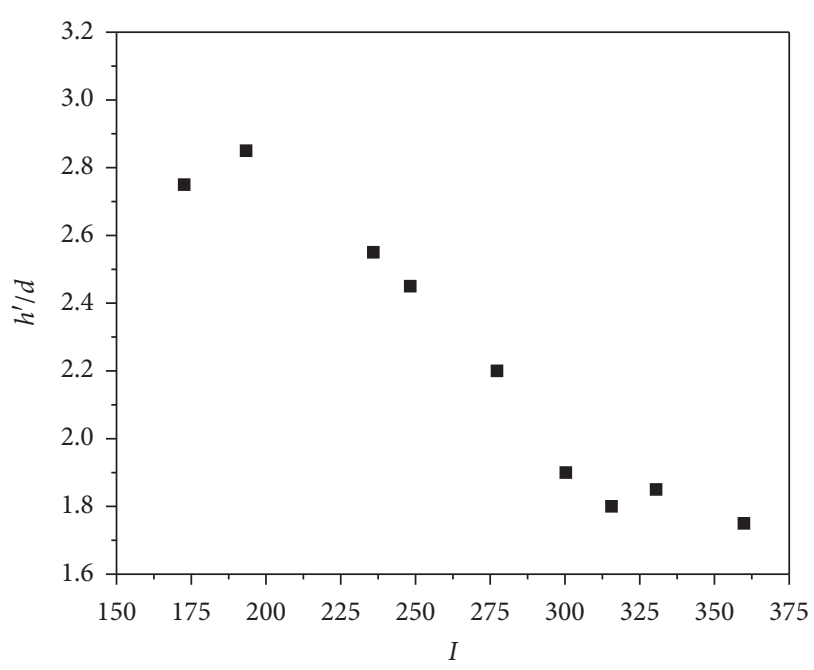

Figure 11: Plot of dimensionless collapse depth as a function of the impact energy factor.

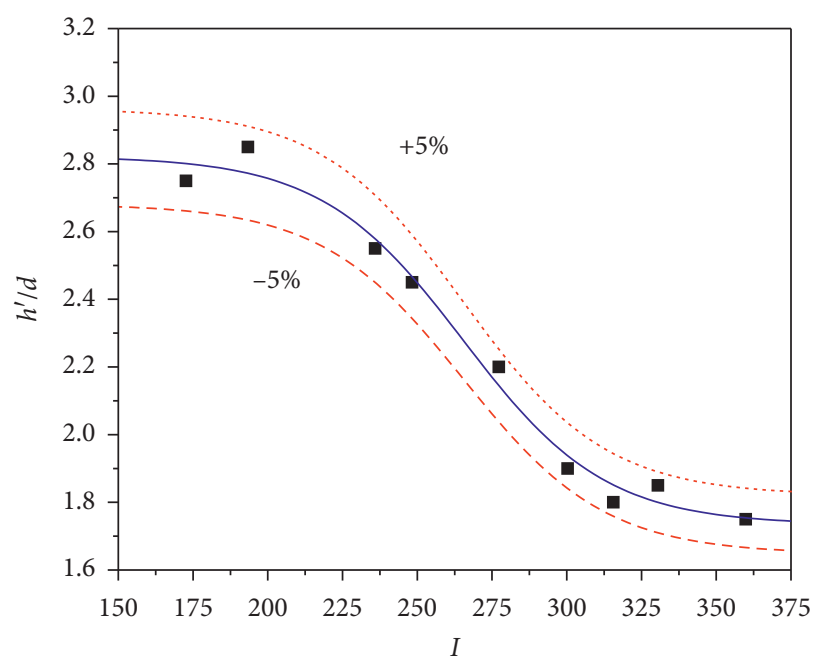

FIgURE 12: Fitting of equation (12) to the experimental results.

TABle 3: Main parameters of the RHT constitutive model.

\begin{tabular}{lc}
\hline Parameter & Value \\
\hline State equation parameter B0 & 1.22 \\
State equation parameter B1 & 1.22 \\
State equation parameter T1 & 100 \\
State equation parameter T2 & 0 \\
Failure surface parameter N & 0.61 \\
Relative shear strength FS* & 0.18 \\
Relative tensile strength FT* & 0.1 \\
Lode angle parameter Q0 & 0.865 \\
Lode angle parameter B & 0.0105 \\
Failure surface parameter A & 1.4 \\
\hline
\end{tabular}

Nevertheless, this equation has limitations and is only applicable to the data range for the materials used in the experiment, which, however, are commonly used in engineering. The strength range of the concrete target is 
TABLE 4: The explosive and its state equation parameters.

\begin{tabular}{lcccccccc}
\hline \multirow{2}{*}{ Density $\mathrm{kg} \cdot \mathrm{m}^{-3}$} & \multirow{2}{*}{ Detonation velocity $\left(\mathrm{m} \cdot \mathrm{s}^{-1}\right)$} & \multirow{2}{*}{$\mathrm{CJ}$ pressure $(\mathrm{GPa})$} & \multicolumn{4}{c}{ JWL state equation parameters } \\
& & & $A(\mathrm{GPa})$ & $B(\mathrm{GPa})$ & $R_{1}$ & $R_{2}$ & $\omega$ & $E(\mathrm{GPa})$ \\
\hline 1200 & 4950 & 6.125 & 52.4 & 0.768 & 4.2 & 1.1 & 0.34 & 8.5 \\
\hline
\end{tabular}

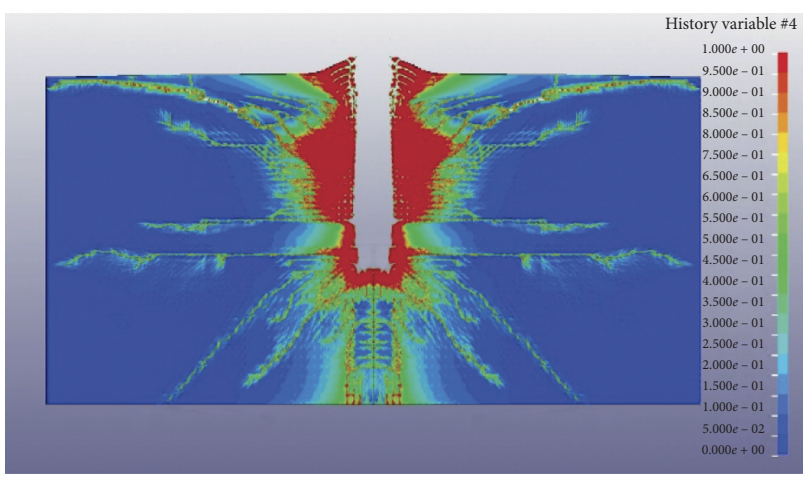

(a)

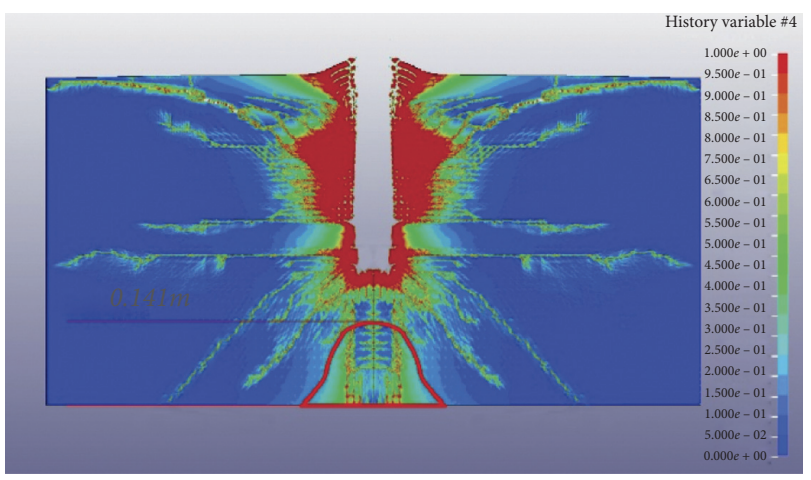

(c)

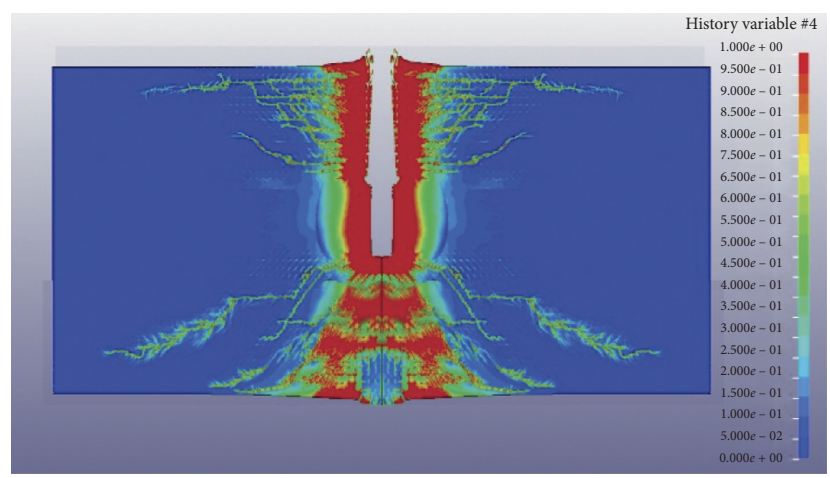

(b)

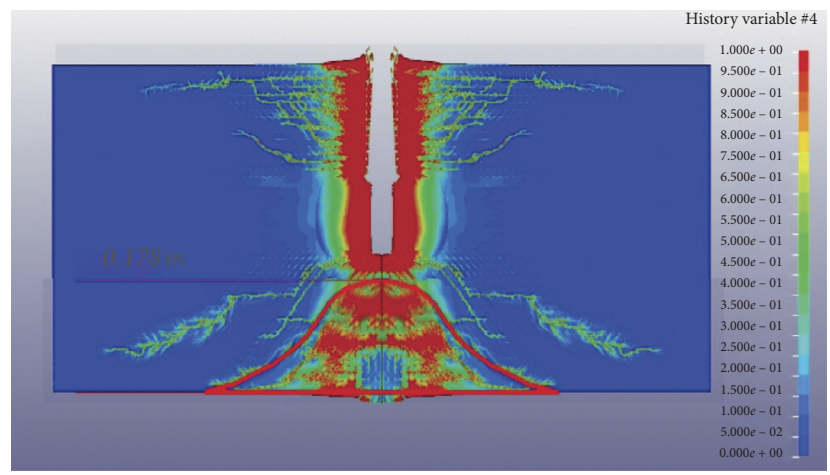

(d)

FIGURE 13: Numerical simulation damage diagram and damage area mark of group 8.

C30-C40, and the projectile that caused the initial penetration damage is normal incidence.

To verify the experimental data and the results of dimensionless analysis, LS-DYNA software and finite-element method were used for numerical simulations. In the numerical simulation, the cylindrical concrete target subjected to penetration and explosion can be simplified into an axisymmetric model. The target and the projectile adopt the shell axisymmetric algorithm unit, and the air and explosive use the ALE2D axisymmetric algorithm unit. The air boundary is set as a nonreflective boundary, and the outer boundary of the target is set as a radial constraint (steel hoop constraint in the experiment). After the penetration, the element node information and stress-strain state of the target are output as the initial conditions for the explosion calculation. Depending on the result of penetration, the location of implosive initiation is determined, and the modeling and calculation of the explosion is carried out. When constructing the concrete RHT constitutive model, the strain rate effect of the material and the characteristics of the compressive damage softening are considered; moreover, the influence of the third invariant of the partial stress tensor on the shape of the failure surface is considered. The elastic limit surface, failure surface, and residual strength surface are used as the control failure surface to describe the initial yield strength, failure strength, and residual strength of the concrete material. The damage evolution of concrete materials can be well modeled. The main parameters of the RHT constitutive model are shown in Table 3.

The air model uses the MAT_NULL model and the EOS_GRUNEISEN state equation. The explosive model uses the MAT_HIGH_EXPLOSIVE_BURN model and the EOS_JWL state equation. The time step for calculating the implosion is 0.67 by default, with a total duration of $5 \mathrm{~ms}$. Table 4 shows the explosive and its state equation parameters. In addition, the projectile is modeled using an elastoplastic model.

Figure 13 shows the numerical simulation damage diagram and damage area mark of group 8 . The red area in Figure 13 is the damaged area of the concrete structure. As the collapse failure is tensile failure, the failure mode of the back surface is layered fracture. To accurately judge the collapse depth of the back surface, the stress-effective destruction layer farthest from the back explosion surface is selected, and the distance between this layer and the back explosion surface is the collapse depth. The numerical 
TABLE 5: Numerical simulation results for collapse damage of concrete targets.

\begin{tabular}{|c|c|c|c|c|c|c|c|}
\hline Group & No. & Penetration speed $(\mathrm{m} / \mathrm{s})$ & Charge aspect ratio & Charge quality (g) & Impact factor & Buried depth (m) & Collapse depth (m) \\
\hline \multirow{2}{*}{1} & $1-1$ & 465.12 & $5: 1$ & 39.25 & 172.68 & 0.212 & 0.105 \\
\hline & $1-2$ & - & $5: 1$ & 39.25 & 0 & 0.212 & 0.163 \\
\hline \multirow{2}{*}{2} & $2-1$ & 489.39 & $5: 1$ & 39.25 & 193.38 & 0.223 & 0.107 \\
\hline & $2-2$ & - & $5: 1$ & 39.25 & 0 & 0.223 & 0.164 \\
\hline \multirow{2}{*}{3} & $3-1$ & 512.75 & $5: 1$ & 39.25 & 209.62 & 0.235 & 0.108 \\
\hline & $3-2$ & - & $5: 1$ & 39.25 & 0 & 0.235 & 0.164 \\
\hline \multirow{2}{*}{4} & $4-1$ & 544.35 & $5: 1$ & 39.25 & 235.93 & 0.256 & 0.114 \\
\hline & $4-2$ & - & $5: 1$ & 39.25 & 0 & 0.256 & 0.166 \\
\hline \multirow{2}{*}{5} & $5-1$ & 558.49 & $5: 1$ & 39.25 & 248.28 & 0.269 & 0.125 \\
\hline & $5-2$ & - & $5: 1$ & 39.25 & 0 & 0.269 & 0.172 \\
\hline \multirow{2}{*}{6} & 6-1 & 589.43 & $5: 1$ & 39.25 & 277.31 & 0.278 & 0.134 \\
\hline & 6-2 & - & $5: 1$ & 39.25 & 0 & 0.278 & 0.175 \\
\hline \multirow{2}{*}{7} & $7-1$ & 612.92 & $5: 1$ & 39.25 & 300.29 & 0.290 & 0.138 \\
\hline & 7-2 & - & $5: 1$ & 39.25 & 0 & 0.290 & 0.176 \\
\hline \multirow{2}{*}{8} & 8-1 & 625.21 & $5: 1$ & 39.25 & 315.60 & 0.305 & 0.141 \\
\hline & $8-2$ & - & $5: 1$ & 39.25 & 0 & 0.305 & 0.178 \\
\hline \multirow{2}{*}{9} & $9-1$ & 643.47 & $5: 1$ & 39.25 & 330.49 & 0.317 & 0.145 \\
\hline & $9-2$ & - & $5: 1$ & 39.25 & 0 & 0.317 & $\geq 0.183$ \\
\hline \multirow{2}{*}{10} & $10-1$ & 671.46 & $5: 1$ & 39.25 & 359.87 & 0.332 & 0.149 \\
\hline & $10-2$ & - & $5: 1$ & 39.25 & 0 & 0.332 & $\geq 0.168$ \\
\hline
\end{tabular}

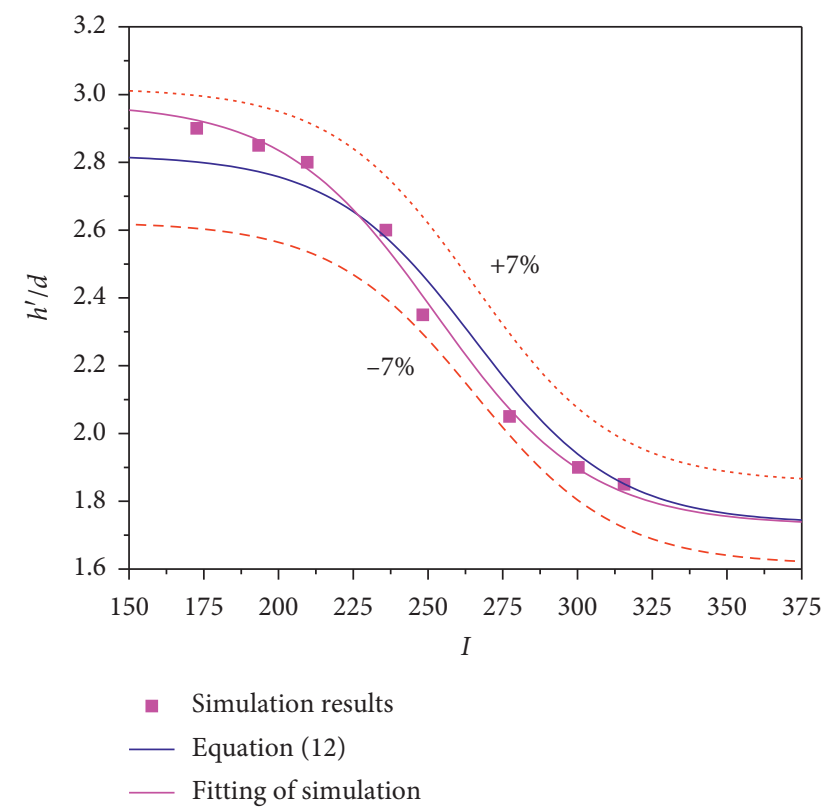

Figure 14: The error between dimensionless analysis and numerical simulation results.

simulation results are shown in Table 5. In the actual experiment, as the concrete structure was not sufficiently vibrated during the casting process, the concrete strength of collapse surface was higher than the strength of the implosive surface. Therefore, the depth of the collapse in the experiment is relatively low, and the depth of the numerical simulation is relatively high. Compare the data with equation (12) and observe that the error between the dimensionless analysis and numerical simulation results does not exceed $7 \%$, and the law of the two results is the same (Figure 14).

\section{Conclusions}

It is of great practical significance to study the effects of the initial penetration damage on the implosion-induced collapse of concrete structures with finite thickness. This study compared the implosion-induced collapse depth of concrete targets with finite thickness under different initial penetration damage through field experiments. The equations obtained by the dimensionless analysis were verified via numerical simulations. The following main conclusions were drawn.

When the impact energy factor increased, the difference in the collapse depth of the concrete targets of the same group decreased, and the decrease rate gradually reduced. When the impact energy factor was small, the initial penetration damage caused more energy overflow due to the small tunnel area, and the implosion-induced collapse effect was significant. Eventually, whether the target was penetrated or the collapse depth gradually stabilized, depending on the relationship between the target thickness and the damage limit, equation (8) was proposed for calculating the influence of the initial penetration damage on the implosion-induced collapse depth of concrete targets and it was fitted to the experimental results, showing good agreement. Then, we derived an equation for calculating the relative collapse depth in the case of the existence of the initial penetration damage. This equation can provide a basis for subsequent experiments to simulate the actual penetration and explosion effects, as well as a reference for the optimal protection design of concrete structures and optimal damage design of penetration-explosion warheads.

In the future, the effects of different length-to-diameter ratios and charges on the implosion-induced collapse effect could be considered in the case of the existence of the initial penetration damage. 


\section{Data Availability}

The data used to support the findings of this study are included within the article.

\section{Conflicts of Interest}

The authors declare that there are no conflicts of interest regarding the publication of this paper.

\section{Acknowledgments}

This study was funded by the National Natural Science Foundation of China (Grant No. 51808553).

\section{References}

[1] G. Wang, S. Zhang, W. Lu et al., "Damage effects of concrete gravity dams subjected to underwater explosion," Journal of Hydraulic Engineering, vol. 46, no. 2, pp. 723-731, 2015.

[2] Q. Zheng, Z. Zhou, Q. Qian et al., "Spallation in protective structures," Chinese Journal of Rock Mechanics and Engineering, vol. 22, no. 8, pp. 1393-1398, 2003.

[3] G. Yang, G. Wang, W. Lu et al., "Damage characteristics of concrete structures under the combined loadings of penetration and explosion," Journal of Central South University, vol. 48, no. 12, pp. 3284-3292, 2017.

[4] J. L. Drake and L. A. Twisdale, Protective Construction Design Manual, (ESL-TR-87-57), Florida: Tyndall Air Force Engineering and Services, Tyndall Air Force Base, FL, USA, 1989.

[5] Y. Fu and Q. Zhang, "Study on blasting crater size model in reinforced concrete," Transactions of Beijing Institute of Technology, vol. 26, no. 9, pp. 761-764, 2006.

[6] J. Li and H. Hao, "Numerical study of concrete spall damage to blast loads," International Journal of Impact Engineering, vol. 68, no. 3, pp. 41-55, 2014.

[7] S. Zhang and G. Wang, "Antiknock performance of concrete gravity dam subjected to underwater explosion," Explosion and Shock Waves, vol. 33, no. 3, pp. 255-262, 2013.

[8] T. Rabczuk, J. Eibl, and L. Stempniewski, "Numerical analysis of high speed concrete fragmentation using a meshfree Lagrangian method," Engineering Fracture Mechanics, vol. 71, no. 4-6, pp. 547-556, 2004.

[9] B. Leng, J. Xu, H. Sun et al., "Numerical simulation of dynamic response of concrete subjected to internal load of blast," Chinese Journal of High Pressure Physics, vol. 23, no. 2, pp. 111-116, 2009.

[10] J. Lai, X. Guo, and Y. Zhu, "Repeated penetration and different depth explosion of ultra-high performance concrete," International Journal of Impact Engineering, vol. 84, pp. 1-12, 2015.

[11] H. Zhang, Z. Duan, L. Yan et al., "Study on the collapse perforation of thick concrete targets under internal explosion," Transactions of Beijing Institute of Technology, vol. 33, no. 5, pp. 441-444, 2013.

[12] U. S. Naval Facilities Engineering Command, Structure to Resist the Effect of Accidental Explosions (TM5-1300), NAVFAC P -397 Design Manual, Alexandria, VA, 1991.

[13] L. I. Sedov, N. M. Friedman, and R. E. Street, Similarity and Dimensional Methods in Mechanics, Mir Publishers, Moscow, Russia, 1982.

[14] J. Tao and K. Zhang, Soil Rock Blasting Similarity Law and Blasting Parameter Optimization, Science Press, Beijing, China, 1998.
[15] P. S. Westine, F. T. Dodge, and W. E. Baker, Similarity Methods in Engineering Dynamics, Hayden Book Co., Rochelle Park, NJ, USA, 1973.

[16] C. W. Lampson, "Explosions in earth," Effects of Impact and Explosion, Office of Technical Services, Department of Commerce, Washington, DC, USA, 1946.

[17] C. B. Morrey, "Underground explosion theory," in Operation Jangle, Office of Technical Services, Department of Commerce, Washington, DC, USA, 1952.

[18] R. M. Hmidt and K. A. Holsapple, "Centrifuge crater scaling experiment II. Material strength effects," Centrifuge Crater Scaling Experiment II Material Strength Effects, 1979.

[19] K. R. Housen, R. M. Schmidt, and K. A. Holsapple, "Crater ejecta scaling laws: fundamental forms based on dimensional analysis," Journal of Geophysical Research, vol. 88, no. B3, pp. 2485-2499, 1983.

[20] P. M. Ghare, D. C. Montgomery, and W. C. Turner, "Optimal interdiction policy for a flow network," Naval Research Logistics Quarterly, vol. 18, no. 1, pp. 37-45, 1971.

[21] K. A. Holsapple and R. M. Schmidt, "On the scaling of crater dimensions: 1. Explosive processes," Journal of Geophysical Research, vol. 85, no. B12, pp. 7247-7256, 1980.

[22] K. A. Holsapple and R. M. Schmidt, "A material-strength model for apparent crater volume," Lunar and Planetary Science Conference Proceedings, vol. 10, pp. 2757-2777, 1979.

[23] R. T. Klinkvort, O. Hededal, and S. M. Springman, "Scaling issues in centrifuge modelling of monopiles," International Journal of Physical Modelling in Geotechnics, vol. 13, no. 2, pp. 38-49, 2013.

[24] V. V. Adushkin and B. D. Khristoforov, "Craters of large-scale surface explosions," Combustion, Explosion, and Shock Waves, vol. 40, no. 6, pp. 674-678, 2004.

[25] R. M. Schmidt, "A centrifuge cratering experiment-development of a gravity-scaled yield parameter," Impact and Explosion Cratering: Planetary and Terrestrial Implications, pp. 1261-1278, 1978.

[26] Q. Qian, N. Sun, and N. Wang, Calculation Principle of Protective Structure, Engineering Academy of PLA, Nanjing, China, 1981. 